\title{
Intracellular MMP-2 Activity in Skeletal Muscle is Associated with Type II Fibers
}

Elin Hadler-Olsen* ${ }^{1}$, Ann Iren Solli ${ }^{1}$, Anne Hafstad ${ }^{1}$, Jan-Olof Winberg ${ }^{1}$ and Lars UhlinHansen $^{1,2}$

${ }^{1}$ Department of Medical Biology, Faculty of Health Sciences, University of Troms $\varnothing$, Norway

${ }^{2}$ Department of Clinical Pathology, University Hospital of North Norway, Troms $\emptyset$, Norway

*Corresponding author:

Dr. Elin Hadler-Olsen

Institute of Medical Biology, Faculty of Health Sciences,

University of Troms $\varnothing, 9037$ Troms $\varnothing$, Norway

Telephone: $+4777620912, \quad$ Fax: +4777644680

E-mail: elin.hadler-olsen@uit.no

Running head: Intracellular MMP-2 in skeletal muscle

Keywords:

- Intracellular MMP-2

- Skeletal muscle fiber

- Type I and type II fiber

Grant information: 


\section{Abstract}

Matrix metalloproteinase 2 (MMP-2) is a proteolytic enzyme implicated in motility, differentiation and regeneration of skeletal muscle fibers through processing of extracellular substrates. Although MMP-2 has been found to be localized intracellularly in cardiomyocytes where the enzyme is thought to contribute to post-ischemic loss of contractility, little is known about intracellular MMP-2 activity in skeletal muscle fibers. In the present study we demonstrate intracellular MMP-2 in normal skeletal muscle by immunohistochemical staining. Immunogold electron microscopic analyses indicated that the enzyme was concentrated in Z-lines of the sarcomers, in the nuclear membrane and in mitochondria. By use of in situ zymography, we found that gelatinolytic activity in muscle fibers was colocalized with immunofluorecent staining for MMP-2. Staining for MMP-9, the other member of the gelatinase group of the MMPs, was negative. The broad-spectrum metalloprotease inhibitor EDTA and the selective gelatinase inhibitor CTT2, but not the cysteine inhibitor E64, strongly reduced the gelatinolytic activity. The intracellular gelatinolytic activity was much more prominent in fast twitch type II fibers than in slow twitch type I fibers, and there was a decrease in intracellular gelatinolytic activity and MMP-2 expression in muscles from mice exposed to high intensity interval training. Together our results indicate that MMP-2 is part of the intracellular proteolytic network in normal skeletal muscle, especially in fast twitch type II fibers. Further, the results suggest that intracellular MMP-2 in skeletal muscle fibers is active during normal homeostasis, and affected by level of physical activity. 


\section{Introduction}

Matrix metalloproteinase 2 (MMP-2), also called gelatinase A, is produced by skeletal muscle fibers during homeostasis and is up-regulated by various physiological and pathological stimuli (Lewis et al., 2000; Carmeli et al., 2005; Carmeli et al., 2010; Dahiya et al., 2011; Alameddine, 2012). Through remodeling of the extracellular matrix (ECM) and by activation of signaling molecules the enzyme can affect motility, differentiation and regeneration of the myofibers (Chen and $\mathrm{Li}, 2009$ ). The enzyme is also reported to be involved in the pathogenesis of muscular dystrophy and atrophy, though the precise mode of action is not well characterized (Fukushima et al., 2007; Kumar and Bhatnagar, 2010; Miyazaki et al., 2011). Although MMP-2 has an N-terminal signal peptide that direct the enzyme for secretion, recent research suggests that it is an inefficient signal causing retention of a substantial portion of the enzyme within cells (Ali et al., 2012). It is a major gelatinolytic enzyme, but can also degrade a number of other matrix and non-matrix substrates (Visse and Nagase, 2003; Rodriguez et al., 2010; Ali et al., 2011). Previously, extracellular functions have been the major focus in MMP-2 research, but recently intracellular variants along with several intracellular putative substrates of this MMP has been reported in some cell types (Cauwe and Opdenakker, 2010; Ali et al., 2011; Hadler-Olsen et al., 2011a). Many of the intracellular substrates have been detected by proteomic analyses, and the precise localization, activity and functions of intracellular MMP-2 is to a large extent unknown. In cardiomyocytes intracellular MMP-2 activity is believed to contribute to the reversible loss of contractile function after acute stress, such as ischemia-reperfusion. In vitro and in vivo studies suggested that this was due to MMP-2 cleavage of troponin I, a central protein in the regulation of muscle contraction, as well as the cytoskeletal protein $\alpha$-actinin (Bolli and Marban, 1999; Wang et al., 2002; Sung et al., 2007b). Other possible MMP-2 substrates in 
cardiomyocytes include desmin, myosin light chain-1 and titin (Ali et al., 2011). Nuclear MMP-2 activity has been associated with apoptosis in several cell types such as in brain neuronal cells, pulmonary endothelial cells and cardiomyocytes (Kwan et al., 2004; Ruta et al., 2009; Yang et al., 2010; Hill et al., 2012).

Due to the similarity in structure and function of cardiac and skeletal muscles, we hypothesised that MMP-2 could be intracellularly localized and active also in skeletal muscle fibers. As MMP-2 is expressed in skeletal muscle during homeostasis (Lewis et al., 2000), we wanted to study putative intracellular MMP-2 localization and activity in normal, healthy muscle fibers. By immunohistochemistry and immunogold analyses we demonstrated MMP-2 intracellularly in compartments not associated with the biosynthetic and secretory pathway in various groups of skeletal muscles in both mice and human. In situ zymography is a method to detect enzyme activity in a tissue section. A quenched, fluorescence-labeled substrate is put on top of a tissue section, and this substrate will be cleaved and fluorescence emitted in areas of the tissue where enzymes with specificity towards the chosen substrate are localized and active, in a time and dose-dependent manner. As sectioning will expose the interior of the vast majority of the cells in the tissue, no permeabilistaion is needed to study intracellular enzymes. By in situ gelatin zymography combined with specific inhibitors and immunofluorescent MMP-2 staining we demonstrated that MMP-2 was active inside the muscle fibers. ATPase staining revealed that the activity was much more prominent in fast twitch type II fibers than in slow twitch type I fibers. Muscles from mice exposed to high intensity interval training showed decreased intracellular MMP-2 expression and gelatinolytic activity compared to their untrained littermates. Taken together our results demonstrate that MMP-2 is intracellularly localized and active inside skeletal muscle fibers during normal homeostasis. The expression and activity of the enzyme is most prominent in fast twitch type 
II fibers and affected by physical activity, but the precise role of the enzyme and the underlying regulatory mechanisms can so far only be speculated.

\section{Materials and Methods}

\section{Cell culturing}

The myoblast $\mathrm{C} 2 \mathrm{C} 12$ cell line was purchased from Sigma-Aldricht (Sigma-Aldich, Steinheim, Germany). The cells were maintained in DMEM containing $4.5 \mathrm{~g} / \mathrm{L}$ glucose (Sigma-Aldricht) and supplemented with $10 \%$ FBS (V/V) (Sigma-Aldricht) in a humidified atmosphere of $5 \% \mathrm{CO}_{2}$ at $37^{\circ} \mathrm{C} .200000$ cells were seeded in 12-well plates, and allowed to attach for 6 hours before changing to a differentiation medium of DMEM containing $4.5 \mathrm{~g} / \mathrm{L}$ glucose supplemented with $3 \%$ horse serum (Sigma-Aldricht). The cells were allowed to differentiate into myocytes for six days before serum-free DMEM was added ( $1 \mathrm{ml} / \mathrm{well})$ after PBS rinsing. Conditioned, serum free medium was harvested after 48 hours, and added HEPES and $\mathrm{CaCl}_{2}$ to a final concentration of $0.1 \mathrm{M}$ and $0.01 \mathrm{M}$ respectively. Detached cells were removed by centrifugation at $1500 \mathrm{x}$ g for 5 minutes at $4^{\circ} \mathrm{C}$, and the supernatant was stored at $-70^{\circ} \mathrm{C}$ until use. After the medium was harvested, cells were washed three times with PBS before cell lysates were prepared in $200 \mu 1$ ice-cold lysisbuffer with proteinase inhibitor $(25 \mathrm{mM}$ Tris- $\mathrm{HCl} \mathrm{pH}$ 7.6, $150 \mathrm{mM} \mathrm{NaCl}, 1 \%$ Triton-X 100, $0.5 \%$ sodium deoxycholate, $0.1 \%$ SDS, $10 \mathrm{mM} \mathrm{CaCl} 2^{-}-2 \mathrm{H}_{2} \mathrm{O}$, Sigmafast proteinase inhibitor cocktail, EDTA free). The cells were scraped, and the lysates collected, sonicated at $4^{\circ} \mathrm{C}$ in a BioRuptor Sonicator (Diagenode s.a, Liege, Belgum) and stored at $-70^{\circ}$ C. Protein content was determined using a Direct Detect Spectrometer (Millipore Corporation, Billerica, MA).

\section{Tissue samples}

Muscle tissue from the gastrocnemius muscle was harvested from 18 weeks old C57BL/6J male mice (Charles River Laboratories, Wilmington, USA). The mice were divided into two 
groups each containing five mice; one which had a normal cage activity (control) and the other where the mice were subjected to five consecutive days of high intensity tread mill running once a day for 60 minutes as previously described (Hafstad et al., 2011). The mice were sacrificed and the gastrocnemius muscle, which contains a mixture of slow twitch and fast twitch muscle fibers were isolated. For mice subjected to high intensity training, the muscles were isolated within minutes after the last round of training. From three untrained mice, samples of the gastrocnemius muscle were also put in Allprotect tissue reagent (Qiagen, Hilden, Germany) for subsequent homogenization and protein extraction. In order to study MMP-2 in muscles from different locations, tongues were harvested from the untrained mice. All mice were treated in accordance with the European Convention for the Protection of Vertebrate Animals for Experimental and Other Scientific Purposes' guidelines on accommodation and care of animals, and the project was approved by the Animal Welfare Committee at the University of Troms $\varnothing$. Samples for light microscopic analyses were fixed in a Zn-based fixative (ZBF) (36.7 $\mathrm{mM} \mathrm{ZnCl}_{2}, 27.3 \mathrm{mM} \mathrm{ZnAc}_{2} \times 2 \mathrm{H}_{2} \mathrm{O}$ and $0.63 \mathrm{mM} \mathrm{CaAc}_{2}$ in $0.1 \mathrm{~mol} / 1$ Tris $\mathrm{pH}$ 7.4), dehydrated and paraffin embedded. From three of the untrained mice, gastrocnemius muscles were also prepared for immunogold electron microscopic analyses by fixation in $4 \%$ formaldehyde in $200 \mathrm{mM}$ Hepes, dehydration and epoxy resin embedding. To assess if MMP-2 staining was similar in muscles from mice and humans, five human ZBFfixed muscular tongue tissue samples were also analyzed. In addition, three human biopsies from the vastus lateralis or biceps muscles were frozen in liquid nitrogen-cooled methylbutane and mounted with Tissue-Tek OCT (Sakura Finetek Europe, Alphen aan den Rijn, The Netherlands) for ATPase staining and gelatin in situ zymography. The human biopsies were collected from the Department of Clinical pathology, University Hospital of North Norway, and all personal data were anonymous. 


\section{Immunohistochemistry, Immunofluorescence and Immunogold Analyses}

Immunohistochemistry was performed on $4 \mu \mathrm{m}$ thick sections of ZBF-fixed, paraffin embedded muscle tissue, from human and mouse tongues as well as the gastrocnemius muscle from mice, with two different primary rabbit antibodies against MMP-2 (NB200-193, Novus Biologicals, Littleton, CO, diluted 1:300 and ab37150, Abcam, Cambridge, MA giving similar results) or a rabbit anti MMP-9 antibody (Ab 38898 Abcam, Cambridge, MA, diluted 1:800). Horseradish peroxidase (HRP)-labelled secondary antibody and diaminobenzidine substrate was used for visualization $\left(\right.$ EnVision $^{+}$system-HRP for rabbit primary antibodies, Dako, Glostrup, Denmark). Staining was performed as previously described (Hadler-Olsen et al., 2010; Solli et al., 2013) on five parallel samples from each location and species. Immunofluorescent staining was performed on the three parallels of mouse tongue and gastrocnemius samples with the primary rabbit MMP-2 antibody from Novus, and visualized with a Cy5 goat anti-rabbit secondary antibody (cat. \# AP187S, Milipore Corporation, Cork, Ireland, diluted 1:200). Fluorescence was studied using a Leica TSC SP5 confocal laser microscope with Leica Application Suite Advanced Fluorescence software (Leica, Wetzlar, Germany). Sections in which the primary antibody was replaced by $5 \%$ normal goat serum were used as negative controls for both immunohistochemical and immunofluorescence analyses.

Electron microscopic immunogold detection of MMP-2 was performed on three different gastrocnemius samples from mice with two different MMP-2 primary rabbit antibodies (NB200-193, Novus Biologicals, diluted 1:200 and LS-B5182, LifeSpan Biosciences Inc., Seattle, WA, diluted 1:5, giving similar results). Prior to primary antibody incubation, unspecific staining was blocked with $0.8 \%$ bovine serum albumin and $0.1 \%$ gelatin from cold water fish skin (Sigma-Aldich, Steinheim, Germany). Gold conjugated antirabbit antibodies (10 $\mathrm{nm}$ ) were used for detection (CMC, Utrecht, Netherlands). 
Sections where the primary antibody was omitted were used as negative controls. The specificity of the antibodies has previously been validated by immunogold staining of cell lines expressing variable amounts of MMP-2 (Solli et al., 2013). Immunogold labelling was studied in a JEOL JEM-1010 transmission electron microscope (JEOL Ltd. Tokyo, Japan) equipped with a Morada digital camera (Olympus Corporation, Hamburg, Germany).

ATPase activity was detected in three different unfixed human samples of the vastus lateralis or biceps muscle at high $\mathrm{pH}$ (9.4) and low pH (4.5) according to standard procedures (Engel and Tice, 1966).

\section{In situ zymography}

Gelatinolytic activity was localized by in situ zymography on five parallel samples of ZBFfixed gastrocnemius (from mouse) and tongue (from human and mouse) muscle samples, as well as on three unfixed human muscle samples from the biceps and vastus lateralis muscle as described earlier (Hadler-Olsen et al., 2010). To verify the contribution of metalloproteinases, $20 \mathrm{mM}$ EDTA was added to the substrate in control sections. As EDTA is a broad spectrum metalloproteinase inhibitor, the selective gelatinase (MMP-2 and MMP-9) inhibitor CTT2 (Pirila et al., 2001; Heikkila et al., 2006) was also used in a $100 \mu \mathrm{M}$ concentration to demonstrate the contribution of MMP-2 to the gelatinolytic activity. Cysteine proteases are known to be major intracellular enzymes; therefore $10 \mathrm{ng} / \mathrm{ml}$ of the cysteine protease inhibitor E-64 (Sigma-Aldirich, Steinheim, Germany) was added to other control sections to evaluate the contribution of such enzymes to the intracellular gelatinolytic activity. The inhibitors CTT2 and E64, applied in the in situ zymography, were tested for quenching, using $4 \mu \mathrm{g} / \mathrm{ml}$ FITC-labeled gelatin (Molecular Probes, Eugene, OR) in the presence or absence of $100 \mu \mathrm{M}$ CTT2 or $10 \mu \mathrm{M}$ E64, in the in situ zymography assay buffer. The fluorescence was determined in a LS50 B Luminescence Spectrometer (Perkin Elmer, Waltham, MA), with excitation 
wavelength of $490 \mathrm{~nm}$, emission wavelength of 520nm and slit width of $10 \mathrm{~nm}$. Neither of the inhibitors could be regarded as quenchers as the fluorescence of the FITC-labeled gelatin was reduced by less than $0.5 \%$.

The level of auto-fluorescence in the tissue was assessed by incubating control sections at $-20^{\circ} \mathrm{C}$. For some sections, in situ zymography was followed by immunofluorescent detection of MMP-2 as described in the previous section. Fluorescence was studied using a Leica TSC SP5 confocal laser microscope with Leica Application Suite Advanced Fluorescence software (Leica, Wetzlar, Germany).

\section{SDS-PAGE gelatin zymography and real-time reverse zymography}

To verify presence of MMP-2 in skeletal muscle by an antibody-independent technique, we also performed SDS-PAGE gelatin zymography on tissue extracts of gastrocnemius muscles from three parallel untrained mice as well as on cell lysates and serum-free conditioned medium of the murine myoblast cell line $\mathrm{C} 2 \mathrm{C} 12$. The muscle tissue that was used for the extraction had been stored in Allprotect tissue reagent (Qiagen, Hilden, Germany) at $-70^{\circ} \mathrm{C}$ until use. Before protein extraction, the Allprotect tissue reagent was rinsed off by PBS, and tissue samples were homogenised as previously described (Hadler-Olsen et al., 2011b). The SDS-PAGE gelatin zymography was with gels containing $0.1 \%(\mathrm{w} / \mathrm{v})$ gelatin and $4 \%$ and $7.5 \%(\mathrm{w} / \mathrm{v})$ polyacrylamide in the stacking and separating gels, respectively performed (Hadler-Olsen et al., 2010). Gelatinolytic activity was evident as cleared regions. As molecular weight standard human pro-MMP9 dimer (225 kDa), pro- and processed MMP-9 monomer $(92 \mathrm{kDa}$ and $88 \mathrm{kDa}$ respectively) and pro- and processed human MMP-2 (72 kDa and $62 \mathrm{kDa}$ respectively) was used. To evaluate the enzyme classes responsible for the observed gelatinolytic activity, two parallel gels were run. One was washed and incubated in buffers containing 
$10 \mathrm{mM}$ EDTA, a metalloproteinase inhibitor, and the other in buffers without inhibitors (control).

In addition, to assess for inhibitors of gelatinolytic enzymes in muscle, the same samples from muscle tissue and myoblast cells were subjected to real-time reverse SDS-PAGE zymography, as previously described (Mathisen et al., 2003). In brief, gels were prepared as for the SDS-PAGE gelatin zymography except that the separating gels contained $13 \%(\mathrm{w} / \mathrm{v})$ polyacrylamide, $0.1 \%$ 2-methoxy-2,4-diphenyl-3(2H)-furanone (MDPF)-labeled gelatin and conditioned medium from the THP-1 monocyte cell line which contains MMP-9 and TIMP-1. This medium was also used as a molecular weight standard. The gel was pre-run for 1 hour before the samples were loaded. During incubation $37^{\circ} \mathrm{C}$, the gels were observed every $30^{\text {th }}$ minute under UV light until a clear contrast between the fluorescent inhibitor bands and the rest of the darkened gel had developed, and photographs were taken.

\section{Mass spectrometry}

To identify unknown gelatinolytic inhibitors detected by reverse SDS-PAGE substrate zymography, muscle tissue extract, $\mathrm{C} 2 \mathrm{C} 12$ serum-free conditioned medium and cell lysate were subjected to non-reducing SDS-PAGE (NuPAGE Novex 4-12\% Bis-Tris gels, Invitrogen, Carlsbad, CA). The gel was stained with Imperial Protein Stain (Thermo Scientific, Rockford, IL), and all defined protein bands with masses between 30 and 10 kDa were cut out. Additionally, bands with masses between 80-60 kDa were cut out. The bands were subjected to mass spectrometric analyses as described in Supplementary file.

\section{Quantification and statistics}


Volocity 3D Image Analysis Software (Perkin Elmer Inc., Waltham, MA) was used for quantification of the fluorescence intensity representing the in situ gelatinolytic activity or MMP-2 staining in muscle fibres from trained and normal control mice. Separating the fiber types was done manually in the Velocity Software using the freehand ROI tool. Areas of the sections where the tissue was disrupted in any way was excluded, otherwise all intact and focused cross sectioned fibers in the field were measured. The strongly fluorescent region in the periphery of the fibers, probably representing the sarcolemma, was included in the quantification. The quantification gives a value of average fluorescence per area in each fiber type. Based on the results from the ATPase and in situ gelatin zymography results described in the result section, fibers with little gelatinolytic activity were interpreted as type I fibers and fibers with strong gelatinolytic activity as type II. The images used were of 20x magnification, and 3-4 different images from one sample provided areas from the whole section to be analysed. To quantify gelatinolytic activity 235 type I and 220 type II fibers from 11 images of 3 different samples were analysed from normal control mice, and 191 type I and 180 type II fibers from 9 images of 3 different samples were analysed from trained mice. To quantify MMP-2 immunofluorescent staining 97 type I and 193 type II fibers from 6 images of 2 different samples were analysed from normal control mice, and 122 type I and 153 type II fibers from 6 images of 2 different samples were analysed from trained mice.

Statistics were performed using SPSS software (Version 21, IBM, Armonk, New York). To analyse the normality of the populations of fiber type I and II in the different groups of mice, Shaphiro-Wilk test was used. Based on the results from this, Independent Samples t-test (for normally distributed populations) or Mann Whitney test (for not normally 
distributed populations) were performed to determine if there were statistically significant differences in fluorescence intensity (in situ zymography and MMP-2 immunofluorescent staining) between type I and type II fibers from normal and trained mice.

\section{Results}

\section{Intracellular MMP-2 localization and activity in skeletal muscle fibers}

To assess intracellular MMP-2 localization in skeletal muscle tissue, tissue samples from different muscle groups in mice and humans were immunohistochemically stained for MMP2. Staining pattern was similar in all muscles stained both in mice (Figure 1A and B, Figure 4 C and D (immunofluorescence)) and humans (Supplementary file Figure S1 A and B). In longitudinally cut muscle fibers, a cross-striated staining pattern as well as staining of the nuclear membranes were seen in many myocytes (Figure 1A, S1A).The staining intensity varied somewhat between individual muscle fibers within a bundle. Cross sectioned muscle fibers also showed variable staining intensity of adjacent muscle fibers (Figure 1B, S1B). A slightly stronger staining was observed in the periphery of many myofibers which may correspond to the endomysium, the sarcolemma and/or mitochondria which are often found in large numbers in the periphery of muscle fibers (Figure 1B). Negative control sections did not reveal any staining (Supplementary file Figure S1C). MMP-9/gelatinase B, the other member of the MMP gelatinase group, was not detected in any of the skeletal muscle fibers (Supplementary file, Figure S1D (mouse) and S1E (Human)).

To further determine the intracellular localization of MMP-2 in the skeletal myofibers, immunogold electron microscopic analyses were performed on sections of the gastrocnemius muscle of mice. This revealed that in many fibers, MMP-2 was concentrated but not exclusively seen in the Z-bands (Figure 1C). In cross sections, MMP-2 could be detected in 
the periphery of the myofibrils, probably in the sarcoplasmic reticulum or in T-tubuli (Figure 1D). MMP-2 labeling was also observed in mitochondria (Figure 1E) as well as along the nuclear membrane (Figure $1 \mathrm{~F}$ ) and some scattered labeling throughout the muscle in unspecified compartments. Negative control sections did not reveal any labeling (Supplementary file Figure S1F).

To verify presence of MMP-2 in muscle by an antibody independent method, which would also detect all other gelatinolytic enzymes in the muscles, tissue extracts of the gastrocnemius muscle from untrained mice as well as serum-free conditioned medium and cell lysate of the murine myoblast cell line $\mathrm{C} 2 \mathrm{C} 12$ were subjected to SDS-PAGE gelatin zymgraphy. The zymography gels revealed strong gelatinolytic bands at $72 \mathrm{kDa}$, corresponding to the mass of MMP-2, from all samples (Figure 2a), as well as a bands of about $75 \mathrm{kDa}$ of unknown nature. Lanes loaded with $\mathrm{C} 2 \mathrm{C} 12$ serum-free, conditioned medium and cell lysate also showed gelatinolytic bands corresponding to processed MMP-2 (about 65 and $60 \mathrm{kDa}$ ). Some of the lanes loaded with muscle extracts revealed a diffuse band of about $110 \mathrm{kDa}$ which may correspond to mouse MMP-9, reported to be somewhat larger than human due to more extensive glycosylation (Masure et al., 1997). In gels where EDTA was added to the washing and incubation buffer, all bands disappeared, confirming that the gelatinolytic bands were produced by metalloproteases (Figure 2b). Mass spectrometry confirmed presence of MMP-2 and also detected MMP-14, MMP-23 and MMP-24 in C2C12 cell lysate (data not shown).

To investigate presence of inhibitors of gelatinolytic enzymes in muscles, the same samples were subjected to real-time reverse gelatin zymography, using MDPF-labeled gelatin in the gels. All samples contained inhibitors with mass corresponding to TIMP-1 (28 kDa) in the THP-1 medium (leukemic monocyte cell line) used as molecular mass standard (Figure 2c). The muscle extracts and myoblast cell lysate produced additional bands with various 
molecular masses. Mass spectrometry analyses detected TIMP-1, TIMP-2 as well as TIMP-3 in the $\mathrm{C} 2 \mathrm{C} 12$ cell lysate (data not shown).

\section{Intracellular MMP-2 was co-localized with gelatinolytic activity}

To investigate if the intracellular MMP-2 was active, in situ gelatin zymography was performed followed by immunofluorescent staining for MMP-2. MMP-2 is a potent gelatinolytic enzyme, and accordingly, intracellular gelatinolytic activity (Figure 3A) was found to be co-localized with MMP-2 staining (Figure 3A-D). In situ gelatin zymography further revealed strong activity in the periphery of muscle fibers, accompanied by punctuated activity inside the fibers in muscles from both mice (Figure $3 \mathrm{~A}$ and E) and human (Supplementary file Figure S2A). There was a distinct difference in staining intensity between adjacent fibers (Figure 3E, S2A). In longitudinally cut muscles (Figure 3A) prominent gelatinolytic activity was seen with the same cross-striated pattern as the MMP-2 staining (Figure 3B). In contrast to the immunohistochemical MMP-2 staining, no distinct gelatinolytic activity was seen in the nuclear membrane, indicating that the enzyme in this location was inactive. Addition of the metalloprotease inhibitor EDTA caused an almost complete inhibition of the gelatinolytic activity (Figure 3F, supplementary file Figure S2B), indicating that metalloproteases were responsible for the observed activity. The selective gelatinase inhibitor CTT2 also showed a strong inhibitory effect (Figure 3G), though not as complete as EDTA, supporting that MMP-2 was active inside the muscle fibers. The cysteine protease inhibitor E-64 had no apparent effect on the activity (Figure 3H). No activity was seen in the freeze-inhibited sections (Supplementary file Figure S2C), indicating that there was no auto-fluorescence in the tissue. 


\section{Fast twitch type II fibers had more intracellular gelatinolytic activity than slow twitch type I fibers}

In the muscle fibers we could see abundant MMP-2 staining and co-localized gelatinolytic activity in normal, healthy muscle fibers, but the intensity of both the staining and the gelatinolytic activity varied among individual fibers in the same muscle bundle. To investigate if this difference in gelatinolytic activity corresponded to muscle fiber type, we performed ATPase staining $(\mathrm{pH} 4.5$ and $\mathrm{pH}$ 9.4) on unfixed, snap-frozen muscle tissue (from the vastus lateralis and biceps muscles) to separate type I slow twitch and type II fast twitch muscle fibers. In situ gelatin zymography was performed on serial sections from the same tissue. As snap-freezing does not preserve the morphology of the tissue as well as ZBF, the images from these experiments doesn't reveal as many details as those from analyses of ZBFfixed samples as shown in the other figures. At pH 9.4 the type II fibers show strong ATPase activity and develop a dark brown color, whereas type I fibers show weak ATPase activity and develop a light brown color. At pH 4.5 the picture is opposite (Engel and Tice, 1966). Comparison of the same areas in ATPase stained sections at $\mathrm{pH} 9.4$ and in consecutive sections subjected to in situ gelatin zymography (Figure 4, upper panel) showed a strong correlation between gelatinolytic activity and fiber type. Type II fibers appeared to have significantly more gelatinolytic activity than type I fibers. This was also the case with ATPase staining at $\mathrm{pH} 4.5$ (Figure 4 lower panel).

\section{Intracellular gelatinolytic activity and MMP-2 expression was reduced by physical exercise}

To investigate whether increased physical exercise could affect the level of intracellular gelatinolytic activity in muscles, in situ gelatin zymography was performed on the gastrocnemius muscle from $\mathrm{C} 57 \mathrm{BL} / 6 \mathrm{~J}$ mice exposed to high intensity tread mill running for 
five consecutive days (Figure 5B) and their untrained littermates (Figure 5A). Quantification of mean fluorescence intensity (Figure 5E) representing gelatinolytic activity showed a statistically significant decrease $(\mathrm{p}<0.001)$ in fluorescence intensity in both type I and type II fibers of mice exposed to high intensity interval training compared to the control littermates. To verify that MMP-2 contributed to this difference in gelatinolytic activity, the gelatinase inhibitor CTT2 was added to control sections. This showed a strong inhibitory effect in both groups (data not shown). Furthermore there was a strong correlation between intensity of MMP-2 immunofluorescent staining and level of gelatinolytic activity (Figure 5A, B, D, E, F). The differences in MMP-2 staining intensity between trained and normal mice were also statistically significant for both type I and type II fibers $(\mathrm{P}<0.001)$.

\section{Discussion}

In this study we show, by immunohistochemistry and immunogold electron microscopy, that MMP-2 is localized intracellularly in compartments other than the secretory pathways in skeletal muscle fibers. We found the enzyme concentrated, but not exclusively expressed in Z-lines of the sarcomere, in mitochondria as well as in the nuclear membrane of normal skeletal muscle fibers, but further studies are warranted to precisely determine the intracellular localizations of MMP-2. Immunohistochemical staining of MMP-2 was colocalized with gelatinolytic activity in the muscle fibers, although there were compartments with MMP-2 staining that did not reveal gelatinolytic activity, such as the nuclear membrane. This indicates that the MMP-2 in this location was inactive. The gelatinolytic activity was almost completely inhibited by the metalloprotease inhibitor EDTA. The selective MMP-2 and MMP-9 inhibitor CTT2 (Heikkila et al., 2006) also had a strong inhibitory effect, although not as complete as EDTA. Immunohistochemical staining for MMP-9, the other member of the MMP gelatinase group, was negative in the muscle fibers. Also SDS-PAGE 
zymography of myoblast cell lysates and conditioned medium showed strong bands corresponding to masses of both pro-and active MMP-2 but no MMP-9 bands. A diffuse band corresponding to the molecular mass of mouse MMP-9 was seen in zymography gels of mouse muscle tissue extracts, and may be from stromal cells such as macrophages which are known to produce MMP-9 constitutively (Vandooren et al., 2013). Thus, our results strongly suggest that MMP-2 was the main active gelatinase within the muscle fibers.

Real-time reverse gelatin zymography revealed that there were inhibitors of gelatinolytic enzymes present in both muscle tissue extracts, muscle cell lysates and in conditioned medium from the muscle cells with masses corresponding to TIMP-1 in the standard. The tissue extracts and cell lysates contained additional inhibitors with various molecular masses. By mass spectrometric analyses TIMP-1, TIMP-2 and TIMP-3 were identified in some of these bands. These results indicate that there are several more MMP-inhibitors present intracellularly or at the cell membrane than in the conditioned medium.

MMP-2 is synthesized with an N-terminal signal sequence that directs the protein for secretion, and it is usually activated by MMP-14 at the cell membrane (Hadler-Olsen et al., 2011a). Lately however, several mechanisms for intracellular recruitment and activation of the enzyme have been revealed. An alternative splice variant of MMP-2 lacking the signal sequence was recently discovered, which may account for some of the intracellular pool of the enzyme (Ali et al., 2012). In addition, the signal sequence of MMP-2 was found to be weak, causing retention of a substantial amount of the signal sequence containing MMP-2 within the cells. These mechanisms of intracellular localization of MMP-2 were demonstrated in various cell types, among them cardiomyocytes (Ali et al., 2012), and may cause intracellular retention of MMP-2 also within skeletal muscle fibers. Another recent publication reported an 
alternative MMP-2 splice variant lacking both the N-terminal signal sequence as well as the inhibitory pro-domain. This truncated and active form of the enzyme was found in the cytosolic and mitochondrial fraction of cardiomyocytes and was induced in response to oxidative stress (Lovett et al., 2012). Upon oxidative stress, full-length MMP-2 has also been found to be activated by peroxynitrite and glutathione through modification of the cysteine residue of the enzymes inhibitory pro-domain in cardiomyocytes (Viappiani et al., 2009). As reactive oxygen and nitrogen species are produced in skeletal muscle during contraction (Balon and Nadler, 1994; Pattwell et al., 2004; Powers et al., 2011), the intracellular MMP-2 activity may be due to the same mode of enzyme activation. There are also studies showing that binding of MMPs to molecules such as SIBLING proteins and the alpha 2 chain of collagen type VI can induce conformational changes that dislocates the pro-domain from the active site giving an active, full-length enzyme (Fedarko et al., 2004; Freise et al., 2009). This suggests that MMP-2 could also be activated by binding to intracellular proteins in the skeletal muscle cells without being proteolytically processed. By mass spectrometry, we detected TIMP-2, MMP-14 and MMP-24 in the myocytes. TIMP-2 and MMP-14 are known activators of MMP-2 (Hadler-Olsen et al., 2011a), and MMP-24 is also believed to be a potent MMP-2 activator (Llano et al., 1999), showing that the myocytes also produce the proteins needed for proteolytic activation of MMP-2.

Along with discoveries of intracellular recruitment and activation mechanisms of MMP-2, a number of putative intracellular substrates have been identified. By processing $\alpha$-actinin-1, desmin, myosin light chain-1, troponin I or titin in cardiomyocytes MMP-2 may contribute in regulation of contractile force (Sung et al., 2007a; Cauwe and Opdenakker, 2010; Ali et al., 2011). In vitro studies have also demonstrated that MMP-2 is able to process several mitochondrial proteins such as heat shock protein $75,-90 \alpha$ and $-90 \beta$ and peroxiredoxin 5, 
linking the enzyme to regulation of protein folding and antioxidant activity (Cauwe and Opdenakker, 2010). Within a muscle bundle, we found that the gelatinolytic activity was much stronger in fast twitch type II fibers than in slow twitch type I fibers as judged by ATPase staining and gelatin in situ zymography of the same tissue. In accordance with our findings, it has previously been reported that muscles with abundance of type II fibers, express more MMP-2 at both mRNA and protein level than muscles containing mainly type I fibers (Carmeli et al., 2005; Carmeli et al., 2010). These previous studies have looked at protein expression in muscle homogenates where the extracellular matrix and blood from the microcirculation might have contributed to the pool of MMP-2. Therefore, the sources of MMP-2, and the precise localization of the enzyme in the muscles cannot be determined from these previous studies. Fast twitch type II fibers have high levels of glycolytic enzymes (Schiaffino and Reggiani, 2011), and a number of these, such as enolase- $\alpha,-\beta$ and $\gamma$, fructosebisphosphate, aldolase $\mathrm{A}$ and $\mathrm{GAPDH}$, are identified as putative intracellular MMP-2 substrates (Cauwe and Opdenakker, 2010). Proteolytic processing of these enzymes by MMP-2, could explain why we detected more gelatinolytic activity in the glycolytic type II fibers than in oxidative type I fibers, and suggest a role of MMP-2 in regulation of carbohydrate metabolism.

We found a statistically significant decrease in intracellular gelatinolytic activity in muscles from mice exposed to high intensity interval training. In contrast to our results, increased levels of MMP-2 in muscles have previously been reported after 10 days of endurance exercise in humans, and after two to eight weeks of high-intensity training in rats (Carmeli et al., 2005; Rullman et al., 2009; Carmeli et al., 2010; Deus et al., 2012) . Furthermore, a study by Yeghiazaryan et al. found gelatinolytic activity in the nucleus of skeletal muscles in rats which increased after 5 days of repeated physical training (Yeghiazaryan et al., 2012). The 
discrepancy between these studies and ours may be due to species differences, as we have studied mouse muscle, and not rat. The relative intensity and duration of the exercise regiments may also contribute to different results, as well as differences in experimental conditions and detection methods. Except the study by Yeghiazaryan et al., these previous studies have not discriminated between intracellular and extracellular MMP-2 in the muscles, so the apparent discrepancy between our results and previous results may be due to increased MMP-2 secretion along with less intracellular retention during high- intensity training. This is in line with studies from cardiomyocytes, where ischemia-reperfusion injuries caused increased MMP-2 levels in the coronary effluents which were accompanied by a depletion of intracellular MMP-2 levels (Cheung et al., 2000). Ischemia-reperfusion injuries in hearts have furthermore been shown to cause a rapid induction of MMP-2 transcription, which may quickly re-establish intracellular MMP-2 levels (Alfonso-Jaume et al., 2006). The reduced levels of MMP-2 and gelatinolytic activity that we measured in muscles from trained mice could therefore be due to the fact that they were sacrificed only minutes after the last round of exercise, before the MMP-2 levels had been reestablished. If contraction induces activation of intracellular MMP-2, this could be a control mechanism to avoid harmful over-activity of the enzyme inside the muscle cell, or due to increased need of extracellular MMP-2 upon muscle contraction.

Taken together our results demonstrate intracellular localization and activity of MMP-2 in skeletal muscle fibers, which is most prominent in type II fast twitch fibers. The amount and activity of the enzyme was affected by physical activity. The specific intracellular functions of the enzyme can so far only be speculated. MMPs have long been regarded as appealing drug targets in various diseases, especially in cancer patients, where broad spectrum MMPinhibitors have been tested in clinical trials. Musculoskeletal pain has been a frequent side 
effect reported after prolonged use of such inhibitors (Zucker et al., 2000; Brinckerhoff and Matrisian, 2002; Coussens et al., 2002), and suggests that MMPs are implicated in muscular physiology. Thus to be able to develop safe and predictably working MMP-inhibitors, and to expand our understanding of the physiology of skeletal muscles, further studies of these new aspects of MMP-2 biology are warranted.

\section{Acknowledgements}

Randi Olsen and Helga Marie Bye are appreciated for their expertise and technical help with the electron microscopic work. We also thank Kenneth Larsen for help with confocal microscopy and image analyzes, Dr. Jack-Ansgar Bruun for help with mass spectrometry analyses and Dr. Sigurd Lindal for providing biopsies of human skeletal muscle. We are also grateful for technical assistance from Eli Berg, Bente Mortensen and Cristiane Jacobsen.

\section{Grant information:}

This work was supported by grants from the North Norwegian Regional Health Authorities, The Norwegian Cancer Association and the Erna and Olav Aakre foundation.

\section{Conflict of Interest}

No conflicts of interest, financial or otherwise, are declared by the authors. 


\section{Reference list}

Alameddine HS. 2012. Matrix metalloproteinases in skeletal muscles: friends or foes? Neurobiol Dis 48:508-18.

Alfonso-Jaume MA, Bergman MR, Mahimkar R, Cheng S, Jin ZQ, Karliner JS , Lovett DH. 2006. Cardiac ischemia-reperfusion injury induces matrix metalloproteinase-2 expression through the AP-1 components FosB and JunB. Am J Physiol Heart Circ Physiol 291:H1838-46.

Ali MA, Chow AK, Kandasamy AD, Fan X, West LJ, Crawford BD, Simmen T , Schulz R. 2012. Mechanisms of cytosolic targeting of matrix metalloproteinase-2. J Cell Physiol 227:3397-404.

Ali MA, Fan X, Schulz R. 2011. Cardiac sarcomeric proteins: novel intracellular targets of matrix metalloproteinase-2 in heart disease. Trends Cardiovasc Med 21:112-8.

Balon TW , Nadler JL. 1994. Nitric oxide release is present from incubated skeletal muscle preparations. J Appl Physiol (1985) 77:2519-21.

Bolli R , Marban E. 1999. Molecular and cellular mechanisms of myocardial stunning. Physiol Rev 79:609-34.

Brinckerhoff CE , Matrisian LM. 2002. Matrix metalloproteinases: a tail of a frog that became a prince. Nat.Rev.Mol.Cell Biol. 3:207-214.

Carmeli E, Beiker R, Maor M , Kodesh E. 2010. Increased iNOS, MMP-2, and HSP-72 in skeletal muscle following high-intensity exercise training. J Basic Clin Physiol Pharmacol 21:127-46.

Carmeli E, Moas M, Lennon S , Powers SK. 2005. High intensity exercise increases expression of matrix metalloproteinases in fast skeletal muscle fibres. Exp Physiol 90:613-9.

Cauwe B , Opdenakker G. 2010. Intracellular substrate cleavage: a novel dimension in the biochemistry, biology and pathology of matrix metalloproteinases. Crit Rev Biochem Mol Biol 45:351-423.

Chen X , Li Y. 2009. Role of matrix metalloproteinases in skeletal muscle: migration, differentiation, regeneration and fibrosis. Cell Adh Migr 3:337-41.

Cheung PY, Sawicki G, Wozniak M, Wang W, Radomski MW, Schulz R. 2000. Matrix metalloproteinase-2 contributes to ischemia-reperfusion injury in the heart. Circulation 101:1833-9.

Coussens LM, Fingleton B , Matrisian LM. 2002. Matrix metalloproteinase inhibitors and cancer: trials and tribulations. Science 295:2387-2392.

Dahiya S, Bhatnagar S, Hindi SM, Jiang C, Paul PK, Kuang S , Kumar A. 2011. Elevated levels of active matrix metalloproteinase- 9 cause hypertrophy in skeletal muscle of normal and dystrophin-deficient mdx mice. Hum Mol Genet 20:4345-59.

Deus AP, Bassi D, Simoes RP, Oliveira CR, Baldissera V, Marqueti Rde C, Araujo HS, Arena R , Borghi-Silva A. 2012. MMP(-2) expression in skeletal muscle after strength training. Int J Sports Med 33:137-41. 
Engel AG, Tice LW. 1966. Cytochemistry of phosphatases of the sarcoplasmic reticulum. I. Biochemical studies. J Cell Biol 31:473-87.

Fedarko NS, Jain A, Karadag A, Fisher LW. 2004. Three small integrin binding ligand N-linked glycoproteins (SIBLINGs) bind and activate specific matrix metalloproteinases. FASEB J 18:7346.

Freise C, Erben U, Muche M, Farndale R, Zeitz M, Somasundaram R, Ruehl M. 2009. The alpha 2 chain of collagen type VI sequesters latent proforms of matrix-metalloproteinases and modulates their activation and activity. Matrix Biol 28:480-9.

Fukushima K, Nakamura A, Ueda H, Yuasa K, Yoshida K, Takeda S , Ikeda S. 2007. Activation and localization of matrix metalloproteinase- 2 and -9 in the skeletal muscle of the muscular dystrophy dog (CXMDJ). BMC Musculoskelet Disord 8:54.

Hadler-Olsen E, Fadnes B, Sylte I, Uhlin-Hansen L, Winberg JO. 2011a. Regulation of matrix metalloproteinase activity in health and disease 1. FEBS J. 278:28-45.

Hadler-Olsen E, Kanapathippillai P, Berg E, Svineng G, Winberg JO , Uhlin-Hansen L. 2010. Gelatin in situ zymography on fixed, paraffin-embedded tissue: zinc and ethanol fixation preserve enzyme activity. J Histochem Cytochem 58:29-39.

Hadler-Olsen E, Winberg JO, Reinholt FP, Larsen T, Uhlin-Hansen L, Jenssen T, Berg E, Kolset SO. 2011b. Proteases in Plasma and Kidney of $\mathrm{db} / \mathrm{db}$ Mice as Markers of Diabetes-Induced Nephropathy. ISRN Endocrinol 2011:832642.

Hafstad AD, Boardman NT, Lund J, Hagve M, Khalid AM, Wisloff U, Larsen TS , Aasum E. 2011. High intensity interval training alters substrate utilization and reduces oxygen consumption in the heart. J Appl Physiol 111:1235-41.

Heikkila P, Suojanen J, Pirila E, Vaananen A, Koivunen E, Sorsa T , Salo T. 2006. Human tongue carcinoma growth is inhibited by selective antigelatinolytic peptides. Int.J.Cancer 118:22022209.

Hill JW, Poddar R, Thompson JF, Rosenberg GA, Yang Y. 2012. Intranuclear matrix metalloproteinases promote DNA damage and apoptosis induced by oxygen-glucose deprivation in neurons. Neuroscience 220:277-90.

Kumar A , Bhatnagar S. 2010. Matrix metalloproteinase inhibitor batimastat alleviates pathology and improves skeletal muscle function in dystrophin-deficient mdx mice. Am J Pathol 177:24860 .

Kwan JA, Schulze CJ, Wang W, Leon H, Sariahmetoglu M, Sung M, Sawicka J, Sims DE, Sawicki G, Schulz R. 2004. Matrix metalloproteinase-2 (MMP-2) is present in the nucleus of cardiac myocytes and is capable of cleaving poly (ADP-ribose) polymerase (PARP) in vitro. FASEB J. 18:690-692.

Lewis MP, Tippett HL, Sinanan AC, Morgan MJ , Hunt NP. 2000. Gelatinase-B (matrix metalloproteinase-9; MMP-9) secretion is involved in the migratory phase of human and murine muscle cell cultures. J Muscle Res Cell Motil 21:223-33.

Llano E, Pendas AM, Freije JP, Nakano A, Knauper V, Murphy G , Lopez-Otin C. 1999. Identification and characterization of human MT5-MMP, a new membrane-bound activator of progelatinase a overexpressed in brain tumors. Cancer Res 59:2570-6. 
Lovett DH, Mahimkar R, Raffai RL, Cape L, Maklashina E, Cecchini G, Karliner JS. 2012. A novel intracellular isoform of matrix metalloproteinase- 2 induced by oxidative stress activates innate immunity. PLoS One 7:e34177.

Masure S, Paemen L, Van Aelst I, Fiten P, Proost P, Billiau A, Van Damme J , Opdenakker G. 1997. Production and characterization of recombinant active mouse gelatinase $B$ from eukaryotic cells and in vivo effects after intravenous administration. Eur J Biochem 244:21-30.

Mathisen B, Lindstad RI, Hansen J, El-Gewely SA, Maelandsmo GM, Hovig E, Fodstad O, Loennechen T , Winberg JO. 2003. S100A4 regulates membrane induced activation of matrix metalloproteinase-2 in osteosarcoma cells. Clin.Exp.Metastasis 20:701-711.

Miyazaki D, Nakamura A, Fukushima K, Yoshida K, Takeda S , Ikeda S. 2011. Matrix metalloproteinase-2 ablation in dystrophin-deficient $\mathrm{mdx}$ muscles reduces angiogenesis resulting in impaired growth of regenerated muscle fibers. Hum Mol Genet 20:1787-99.

Pattwell DM, McArdle A, Morgan JE, Patridge TA, Jackson MJ. 2004. Release of reactive oxygen and nitrogen species from contracting skeletal muscle cells. Free Radic Biol Med 37:1064-72.

Pirila E, Maisi P, Salo T, Koivunen E, Sorsa T. 2001. In vivo localization of gelatinases (MMP-2 and -9) by in situ zymography with a selective gelatinase inhibitor. Biochem.Biophys.Res.Commun. 287:766-774.

Powers SK, Talbert EE , Adhihetty PJ. 2011. Reactive oxygen and nitrogen species as intracellular signals in skeletal muscle. J Physiol 589:2129-38.

Rodriguez D, Morrison CJ , Overall CM. 2010. Matrix metalloproteinases: what do they not do? New substrates and biological roles identified by murine models and proteomics. Biochim Biophys Acta 1803:39-54.

Rullman E, Norrbom J, Stromberg A, Wagsater D, Rundqvist H, Haas T , Gustafsson T. 2009. Endurance exercise activates matrix metalloproteinases in human skeletal muscle. J Appl Physiol 106:804-12.

Ruta A, Mark B, Edward B, Jawaharlal P , Jianliang Z. 2009. Nuclear localization of active matrix metalloproteinase- 2 in cigarette smoke-exposed apoptotic endothelial cells. Exp.Lung Res. 35:59-75.

Schiaffino S , Reggiani C. 2011. Fiber types in mammalian skeletal muscles. Physiol Rev 91:1447531.

Solli AI, Fadnes B, Winberg JO, Uhlin-Hansen L , Hadler-Olsen E. 2013. Tissue- and Cell-Specific Co-localization of Intracellular Gelatinolytic Activity and Matrix Metalloproteinase 2. J Histochem Cytochem.

Sung MM, Schulz CG, Wang W, Sawicki G, Bautista-Lopez NL, Schulz R. 2007a. Matrix metalloproteinase-2 degrades the cytoskeletal protein alpha-actinin in peroxynitrite mediated myocardial injury. J.Mol.Cell Cardiol. 43:429-436.

Sung MM, Schulz CG, Wang W, Sawicki G, Bautista-Lopez NL, Schulz R. 2007b. Matrix metalloproteinase-2 degrades the cytoskeletal protein alpha-actinin in peroxynitrite mediated myocardial injury. J Mol Cell Cardiol 43:429-36. 
Vandooren J, Van den Steen PE , Opdenakker G. 2013. Biochemistry and molecular biology of gelatinase B or matrix metalloproteinase-9 (MMP-9): the next decade. Crit Rev Biochem Mol Biol 48:222-72.

Viappiani S, Nicolescu AC, Holt A, Sawicki G, Crawford BD, Leon H, van MT , Schulz R. 2009. Activation and modulation of $72 \mathrm{kDa}$ matrix metalloproteinase-2 by peroxynitrite and glutathione. Biochem.Pharmacol. 77:826-834.

Visse R , Nagase H. 2003. Matrix metalloproteinases and tissue inhibitors of metalloproteinases: structure, function, and biochemistry. Circ Res 92:827-39.

Wang W, Schulze CJ, Suarez-Pinzon WL, Dyck JR, Sawicki G, Schulz R. 2002. Intracellular action of matrix metalloproteinase- 2 accounts for acute myocardial ischemia and reperfusion injury. Circulation 106:1543-1549.

Yang Y, Candelario-Jalil E, Thompson JF, Cuadrado E, Estrada EY, Rosell A, Montaner J, Rosenberg GA. 2010. Increased intranuclear matrix metalloproteinase activity in neurons interferes with oxidative DNA repair in focal cerebral ischemia. J Neurochem 112:134-49.

Yeghiazaryan M, Zybura-Broda K, Cabaj A, Wlodarczyk J, Slawinska U, Rylski M, Wilczynski GM. 2012. Fine-structural distribution of MMP-2 and MMP-9 activities in the rat skeletal muscle upon training: a study by high-resolution in situ zymography. Histochem Cell Biol 138:75-87.

Zucker S, Cao J , Chen WT. 2000. Critical appraisal of the use of matrix metalloproteinase inhibitors in cancer treatment. Oncogene 19:6642-50. 


\section{Figure Captions}

Figure 1: Intracellular MMP-2 localization

Representative images from at least four independent experiments of immunohistochemical MMP-2 staining of longitudinally (A) and transversely (B) cut muscle fibers in mouse tongue. MMP-2 staining is seen as brown color, whereas nuclei are stained blue by hematoxylin. In CF representative images of immunogold electron microscopic MMP-2 staining of mouse gastrocnemius muscle is shown, MMP-2 staining is seen as dark spots. Figure 1C demonstrates concentration of the enzyme in Z-bands (Z) in cross section, in (D) labeling is seen surrounding myofibrils, image (E) shows concentration of labeling in mitochondria (M) and image $(\mathrm{F})$ at the nuclear membrane.

Figure 2

(A) Representative images of at least three independent experiments of SDS-PAGE gelatin zymography gels of muscle cell extracts (ME) left panel, serum-free, conditioned medium (M) of the myoblast cell line $\mathrm{C} 2 \mathrm{C} 12$ in the middle panel, and cell lysates of the $\mathrm{C} 2 \mathrm{C} 12$ cell line in the right panel. Molecular weight standard (St) contains pro- and processed MMP-9 (92 and $82 \mathrm{kDa}$ respectively) and pro- and processed MMP-2 (72 and $62 \mathrm{kDa}$ respectively). Gelatinolytic enzymes are seen as clear bands on the gel. (B) To assess contribution of metalloproteases to the gelatinolytic activity, parallel gels were run without (left panel) and with (right panel) $10 \mathrm{mM}$ EDTA in the washing and incubation buffer. (C)The same samples were also subjected to real-time reverse gelatin zymography in a polyacrylamide gel with copolymerized gelatin and MMP-9, to analyze presence of inhibitors of gelatinolytic enzymes. Inhibitors are seen as light bands against a darker background. MMP-2 and MMP-9 are seen 
as distinct dark bands at 92 and $72 \mathrm{kDa}$. Standard (St) contains TIMP-1 in addition to MMP-9 and MMP-2 as described above.

Figure 3: Gelatinolytic activity and co-localized MMP-2 staining Representative images of longitudinally cut muscle fibers from mouse tongue subjected to gelatin in situ zymography without inhibitors (A), immunofluorescent staining for MMP-2 (B), nuclear staining with DAPI (C) and overlay of A-C (D). Gelatinolytic activity is seen as green fluorescence, MMP-2 is seen as red fluorescence, nuclei are stained blue by DAPI and co-localization of gelatinolytic activity and MMP-2 staining is seen as yellow color on the overlay picture (D). Representative images of in situ gelatin zymography from at least three independent experiments of muscle fibers from gastrocnemius muscle of mouse in cross section without any inhibitors (E), with 20 mM EDTA (F), with $100 \mu \mathrm{M} \mathrm{CTT2} \mathrm{(G)} \mathrm{and}$ with10 $\mu \mathrm{g} / \mathrm{ml}$ E-64 (H). Gelatinolytic activity is seen as green fluorescence, whereas nuclei are labeled blue by DAPI.

Figure 4: ATPase staining and gelatinolytic activity

In the upper panel a representative image of ATPase staining at $\mathrm{pH} 9.4$ in a section from the vastus lateralis muscle from human is shown, as well as gelatinolytic activity, demonstrated by in situ gelatin zymography, from a sequential section of the same muscle sample. At $\mathrm{pH}$ 9.4 ATPase activity is seen as dark brown staining in fast twitch type II muscle fibers, whereas slow twitch type I fibers have a light brown color. In the lower panel a representative image of ATPase staining at $\mathrm{pH} 4.5$ in a section from the vastus lateralis muscle from another human is shown, as well as gelatinolytic activity, demonstrated by in situ gelatin zymography, from a sequential section of the same muscle sample. At $\mathrm{pH} 4.5$ ATPase activity is seen as light brown staining in fast twitch type II muscle fibers, whereas slow twitch type I fibers 
have a dark brown color. In both upper and lower panel, gelatinolytic activity is seen as green fluorescence, whereas nuclei are stained blue by DAPI. Corresponding fast twitch type II fibers are labeled with the same numbers in pictures of ATPase staining and gelatinolytic activity in both panels.

Figure 5: Gelatinolytic activity and MMP-2 staining in gastrocnemius muscle from normal, and trained mice

Representative images of gelatinolytic activity (A, B) and MMP-2 staining (C, D) in gastrocnemius muscle from normal control mice (A, C), and mice exposed to five days of high intensity interval training (B, D). Gelatinolytic activity is seen as green fluorescence and MMP-2 is seen as red fluorescence. Histogram showing quantification of differences in fluorescence intensity, representing gelatinolytic activity from in situ zymography (E), or MMP-2 staining (F) between normal, trained and db/db mice. Fiber type I and II are analyzed separately and the results are presented by means. Error bars indicate $95 \% \mathrm{CI}$, and * indicate $\mathrm{p}<0.001$. 


\section{Supplementary file}

\section{Materials and methods}

\section{Mass spectrometry}

To identify unknown gelatinolytic inhibitors detected by reverse SDS-PAGE substrate zymography, muscle tissue extract, $\mathrm{C} 2 \mathrm{C} 12$ serum-free conditioned medium and cell lysate were subjected to non-reducing SDS-PAGE (NuPAGE Novex 4-12\% Bis-Tris gels, Invitrogen, Carlsbad, CA). The gel was stained with Imperial Protein Stain (Thermo Scientific, Rockford, IL), and all defined protein bands with masses between 30 and 10 kDa were cut out. Additionally, bands with masses between 80-60 kDa were cut out. Gel pieces were subjected to in gel reduction, alkylation, and tryptic digestion using $6 \mathrm{ng} / \mu \mathrm{l}$ trypsin (V511A, Promega, Wisconsin, USA)(Shevchenko et al., 1996). OMIX C18 tips (Varian, Inc., Palo Alto, CA, USA) was used for sample clean-up and concentration. Peptide mixtures containing $0.1 \%$ formic acid were loaded onto a Thermo Fisher Scientific EASY-nLC1000 system and EASY-Spray column (C18, $2 \mu \mathrm{m}, 100 \AA$ A $50 \mu \mathrm{m}, 15$ $\mathrm{cm}$ ). Peptides were fractionated using a $2-100 \%$ acetonitrile gradient in $0.1 \%$ formic acid over $50 \mathrm{~min}$ at a flow rate of $250 \mathrm{nl} / \mathrm{min}$. The separated peptides were analyzed using a Thermo Scientific Q-Exactive mass spectrometer. Data was collected in data dependent mode using a Top10 method. The raw data was processed using the Proteome Discoverer 1.4 software. The fragmentation spectra were searched against the Swissprot SwissProt_2011_12 database using an in-house Mascot server (Matrix Sciences, UK). Peptide mass tolerances used in the search were $10 \mathrm{ppm}$, and fragment mass tolerance was 0.02 Da. Peptide ions were filtered using a false discovery rate (FDR) set to $2 \%$ for peptide identifications. 


\section{Supplementary Figure captions}

Figure S1:

Representative images of immunohistochemimal staining for MMP-2 in human tongue muscle cut longitudinally (A) and cross sectioned (B). MMP-2 staining is seen as brown color whereas nuclei are stained blue by hematoxylin. Representative image of negative control section for immunohistochemical MMP-2 staining where the primary MMP-2 antibody was replaced by $1.5 \%$ normal goat serum is shown in C. Representative images of immunohistochemical staining for MMP-9 in tongue muscles from mouse (D) and human (E). MMP-9 staining is seen as brown color, whereas nuclei are stained blue by hematoxylin. Representative image of negative control of MMP-2 immunogold labeling of mouse gastrocnemius muscle where the primary antibody was omitted is shown in F.

Figure S2

Representative images of in situ gelatin zymography of muscle fibers from human tongue without any inhibitors (A) and with $20 \mathrm{mM}$ EDTA (B). A control section incubated at $-20^{\circ} \mathrm{C}$ is shown in C. Gelatinolytic activity is seen as green fluorescence, whereas nuclei are labeled blue by DAPI. 
Figure 1
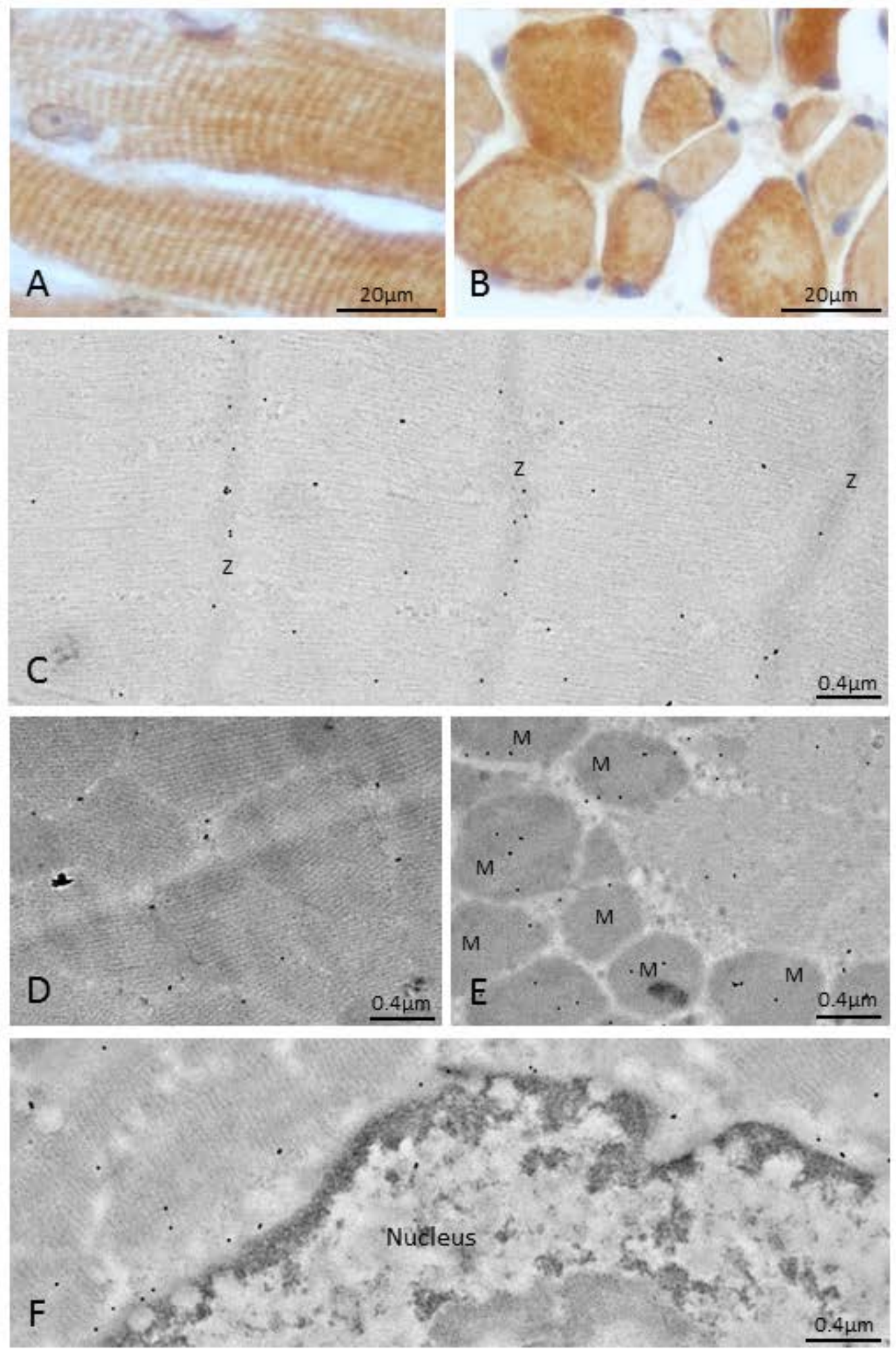
Figure 2

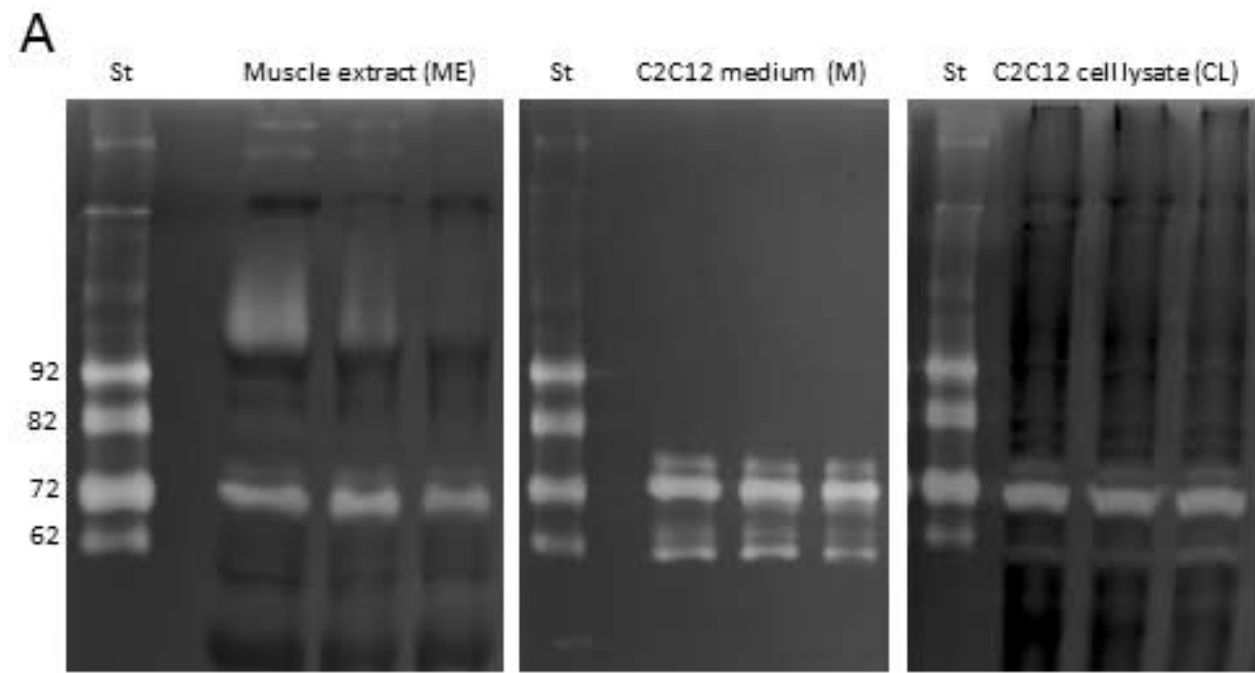

B

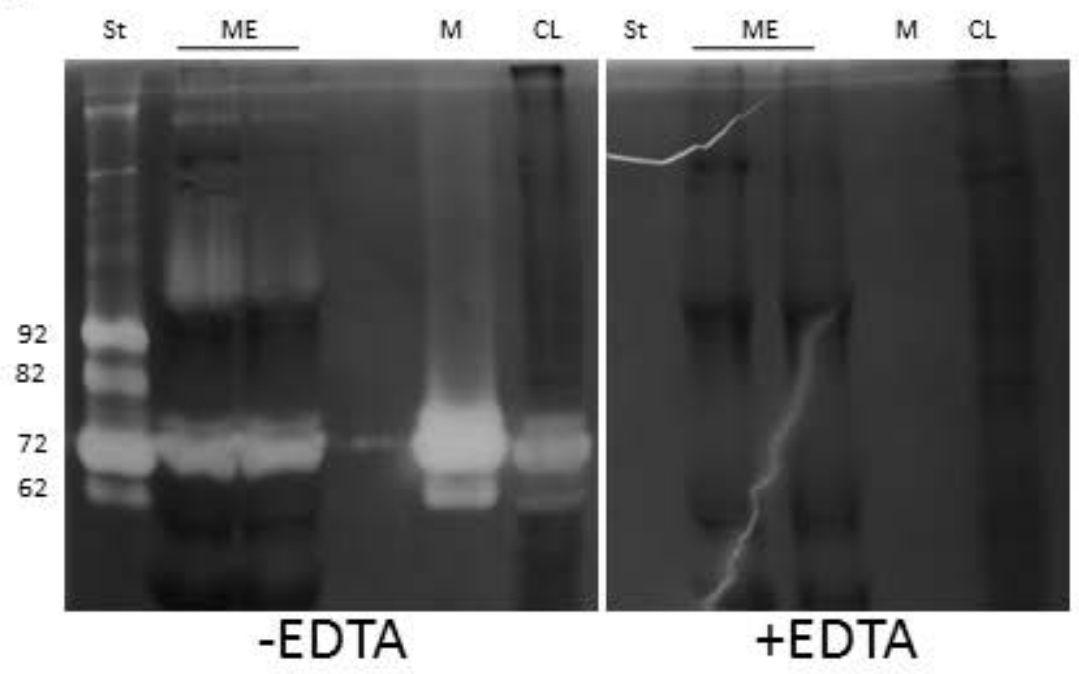

C

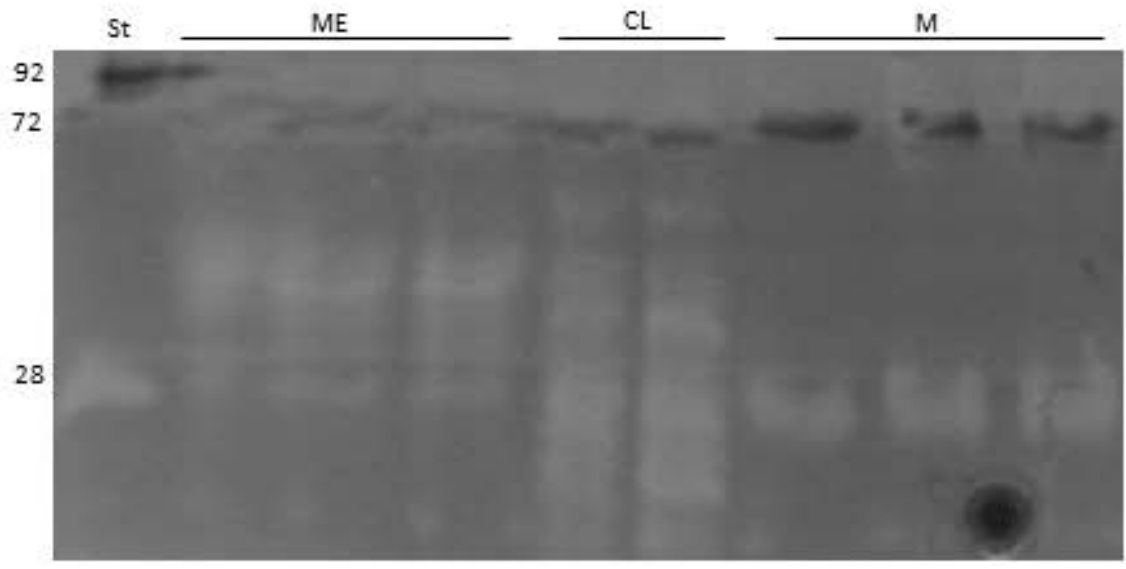


Figure 3
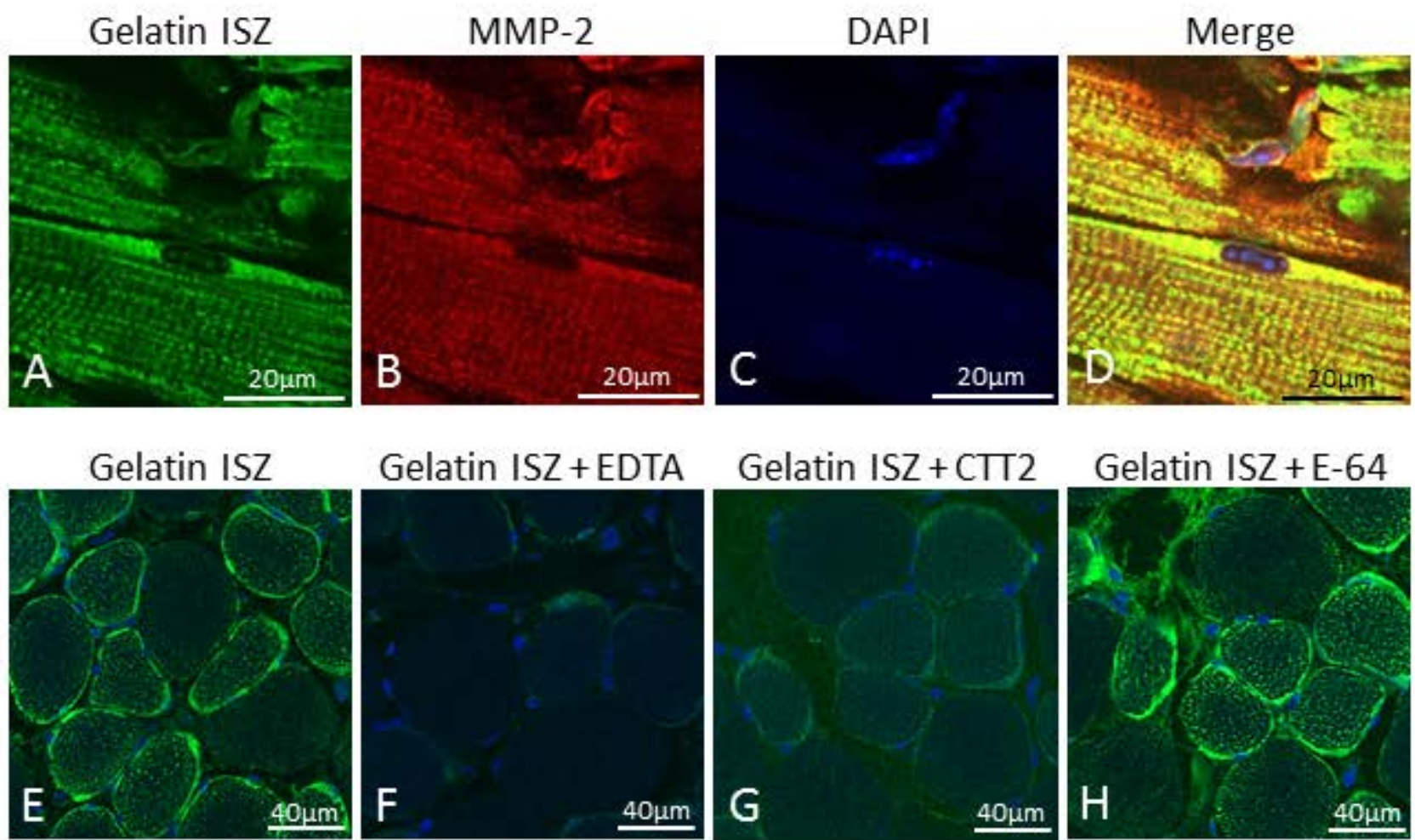
Figure 4
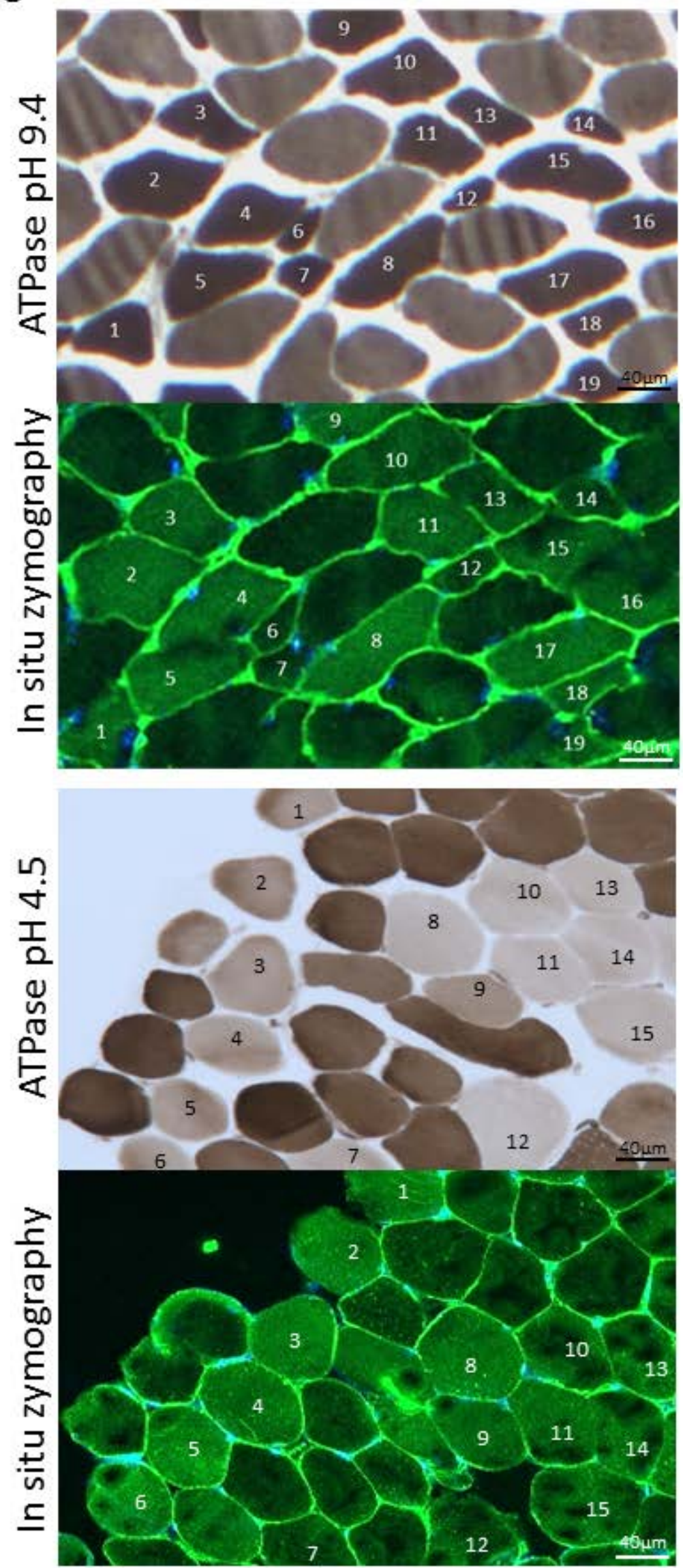


\section{Figure 5}
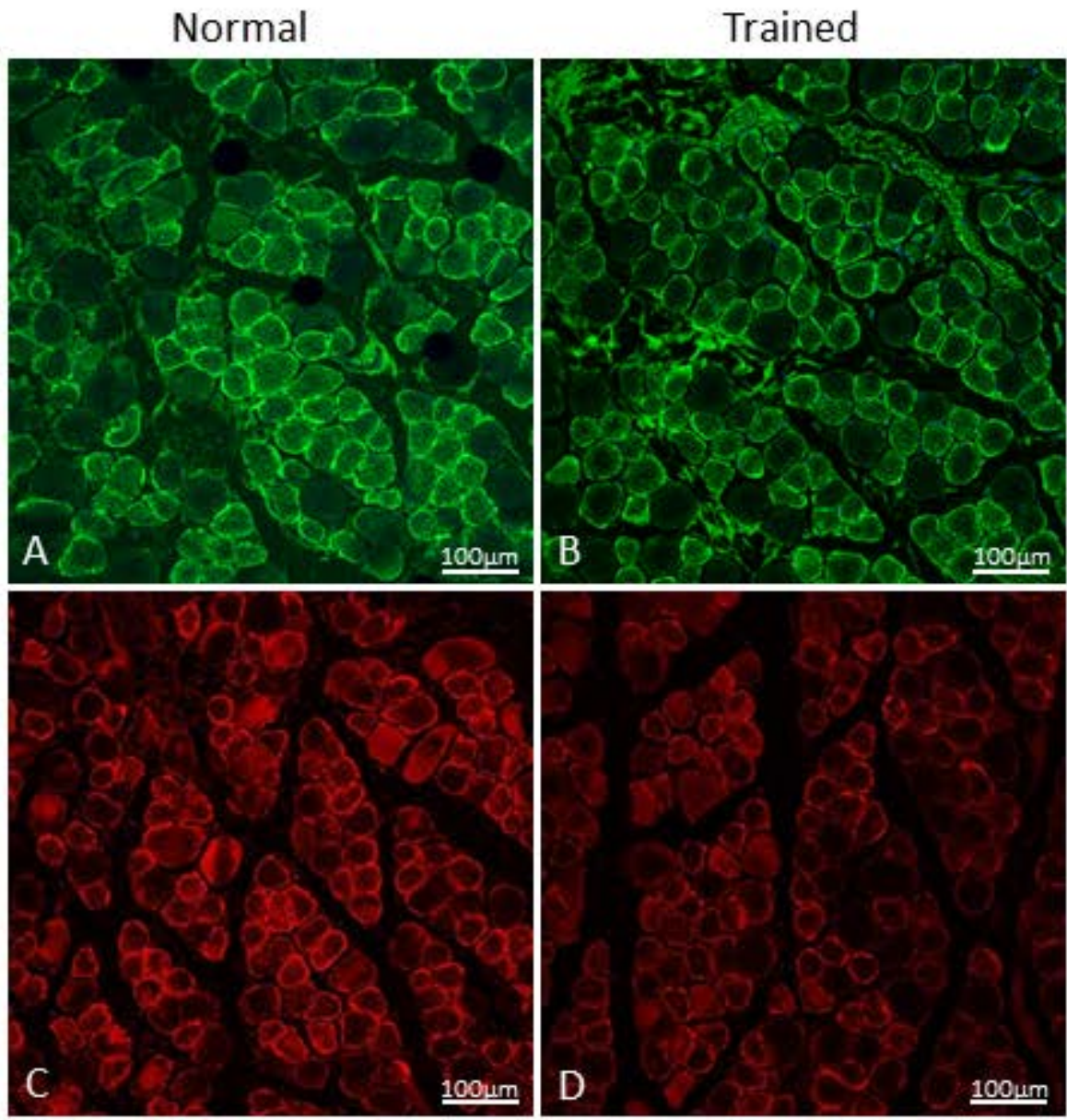

E

F MMP-2 immunofluorescense
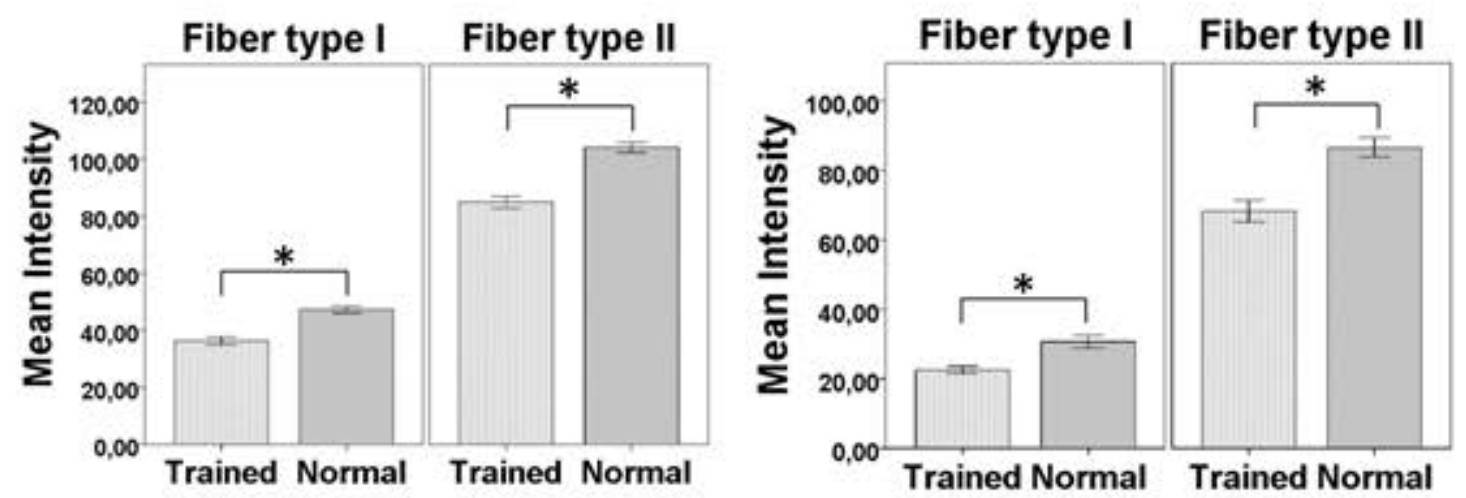
Supplementary file

Figure S1
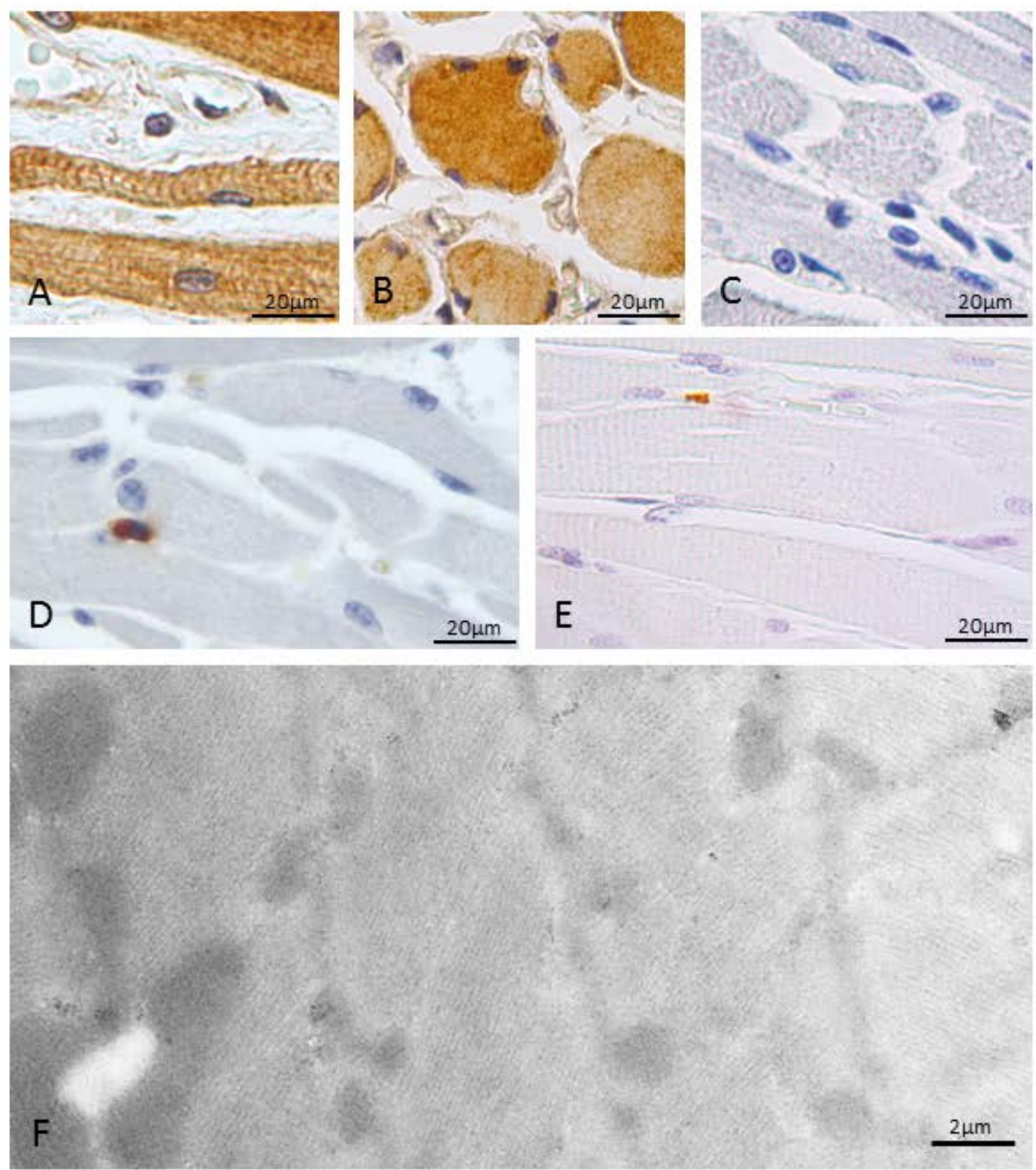
Figure S2
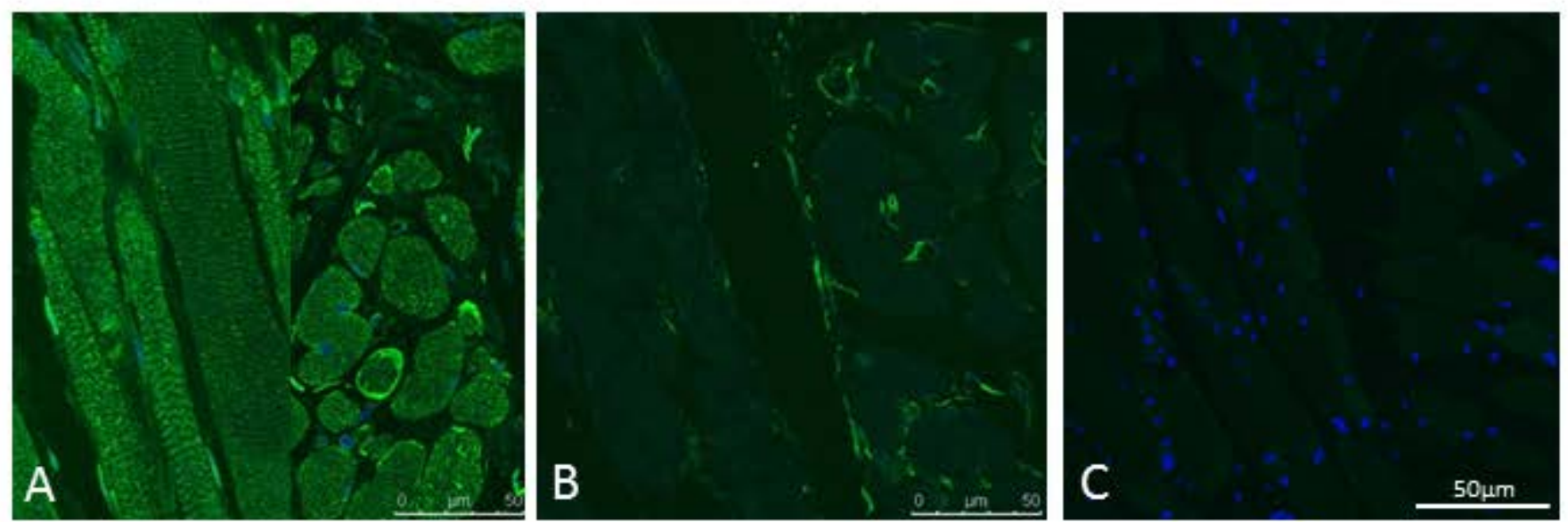